Research Article

Cheng-feng Yue, Yong-bo Wu, and Zhi-lie Tang*

\title{
Visual Analysis of Cylindrically Polarized Light Beams' Focal Characteristics by Path Integral
}

https://doi.org/10.1515/phys-2019-0040

Received Sep 10, 2018; accepted May 10, 2019

\begin{abstract}
To analyze the focal characteristics of cylindrically polarized beams, a visual analysis method is proposed. As known, the focal field can be described by three mutually perpendicular components, each one is the total contribution of all parts of the incident beams. For each component of all contributing parts we apply path integral method, then from the path integral curves extract focal field properties immediately, such as polarization state or intensity distribution. The analysis process of PI is visual and more understandable, and has more powerful information extraction function, which is also helpful for the design of special filtering pupil.
\end{abstract}

Keywords: Visual analysis, Cylindrical polarized, Focal characteristics, Path integral

PACS: 42.25.Bs, 42.15.Dp, 42.25.Fx, 42.25.Hz

\section{Introduction}

Over the years, the special focal characteristics of cylindrically polarized (CP) beams have attracted much interest [1-13]. CP beams are also referred as radially polarized (RP) and azimuthally polarized (AP) beams. The special intensity distribution of CP beams on the focal plane has many wide applications. For example, the donut intensity distribution of AP beams can be used in particle trapping [14-17]. The tight focal spot of the longitudinal field of $R P$ beams is useful in improving the resolution of confocal microscopy [18-21] and optical data storage [22, 23].

The basic theory by which focused polarized beams can be analyzed was originally described by Richards and

\footnotetext{
*Corresponding Author: Zhi-lie Tang: Physics and telecommunication engineering college of South China Normal University Guangdong-guangzhou, 510006, China; Email: tangzhl@scnu.edu.cn Cheng-feng Yue, Yong-bo Wu: Physics and telecommunication
engineering college of South China Normal University GuangdongCheng-feng Yue, Yong-bo Wu: Physics and telecommunication
engineering college of South China Normal University Guangdongguangzhou, 510006, China
}

Ә Open Access. (c) 2019 C.-F. Yue et al., published by De Gruyter. (cc) BY License
Wolf [1, 2] in 1959 and remains the basic principle of vector beams' focal problems until now. However, the role of every part that contributes to the focal field cannot be evaluated easily using only algebraic integral calculation, and the results are somewhat puzzling. To overcome these drawbacks, a visual analysis method was proposed that focuses on the path integral(PI) curves rather than purely on mathematical equations.

Contrary to algebraic integral, the advantage of PI is its intuition and understandability. The use of PI will be beneficial to the analysis of vector beams focusing problems, and the combination of PI and algebraic calculation can extract more information, more easily and comprehensively.

\section{Theory of PI}

Initially, the concept of PI was proposed in the 1940s by American physicist Feynman [24] for expressing quantum amplitude. Later Feynman used PI to illustrate the superposition of coherent light. For revealing the essence of Fermat's principle, Feynman described the mirror reflection phenomenon by PI method. He divided the mirror into many small pieces as shown in Figure 1(a). According to the diffraction theory, every ray of light reflected from $\mathrm{S}$ point through the mirror to $\mathrm{P}$ point may hold, and their amplitudes are same, but the phases are different because of the different paths. So, Feynman represented them with arrows in different directions, as shown in Figure 1(b). The light field of point $P$ denoted by vector $\overrightarrow{A B}$ is the superposition of all these vectors, as shown in Figure 1(c). Then by analyzing Figure 1(c), Feynman validated Fermat's principle.

\section{Basic focal theory of CP beams}

Figure 2 shows the geometry of the focal system. According to Richards and Wolf theory, the electric field at point Q generated by RP and AP beams near point $\mathrm{P}$ can be written 


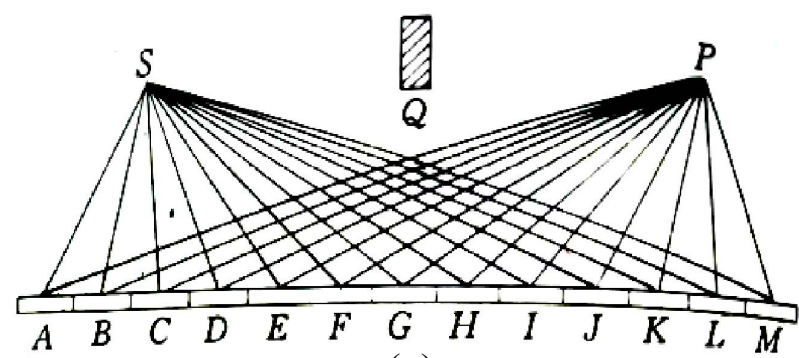

(a)

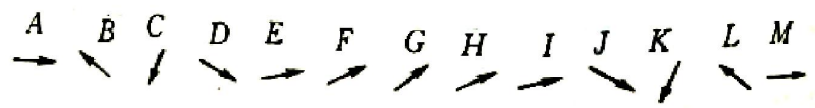

(b)

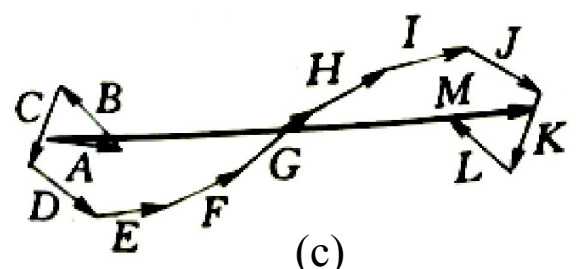

Figure 1: The illustration of Fermat's Path Integral principle : the image of mirror reflection

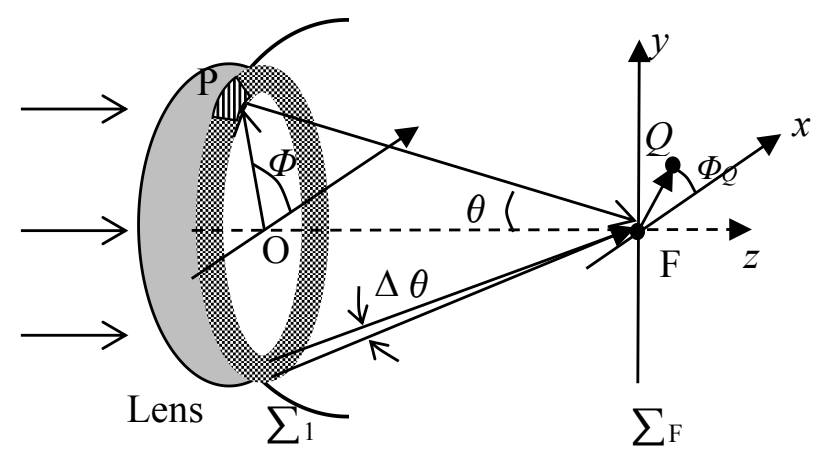

Figure 2: Focal system geometry. $\sum_{1}$ is the equal phase spherical surface just after the lens. $\sum_{F}$ is the focal plane. $O$ is the center of the lens. $\mathrm{F}$ is the focus. $\mathrm{P}$ and $\mathrm{Q}$ are points on $\sum_{1}$ and $\sum_{F}$, respectively, and $\Phi$ and $\Phi_{Q}$ are the azimuth angles of P and $Q$ respectively. $\theta$ is the diffraction angle of light PF. $\Delta \theta$ is the angle width of the annular beams

as

$$
\begin{aligned}
& d \tilde{\vec{E}}_{P Q}=\frac{\text { if } A_{0}(\theta)}{\lambda} \sqrt{\cos \theta} \sin \theta \\
& \times\left(\begin{array}{c|cc}
R P & A P & \\
\cos \theta \cos \varphi & -\sin \varphi & \hat{x} \\
\cos \theta \sin \varphi & \cos \varphi & \hat{y} \\
\sin \theta & 0 & \hat{z}
\end{array}\right) e^{i k_{0} \rho_{Q} \sin \theta \cos \left(\varphi_{Q}-\varphi\right)} d \theta d \varphi
\end{aligned}
$$

where $\varphi$ denotes the azimuth relative to the $\mathrm{x}$-axis. $\theta$ is the semi-angular aperture of the annular beams. $A_{0}(\theta)$ is the light beams' amplitude distribution, which is supposed to be constant $A_{0} . \lambda$ and $f$ are the wavelength and focal length. $\rho_{Q}$ is the distance of point $Q$ to focus F. $\varphi_{Q}$ is the azimuth of $Q$ to the $x$-axis.

A clear circular aperture can always be treated as the sum of many annular rings with different radius; thus, the focal properties of a narrow (with angular width $\Delta \theta<<$ $\pi / 2$ ) annular beams are studied here. In fact, using large numerical annular beams to achieve a tight focus has been proposed by many papers [25-27]. Here suppose the annular beams numerical aperture(N.A) is 1 .

\section{RP beams' focal properties}

\subsection{Transverse focal properties}

The annular beams shown in Figure 2 can be separated into a number of small facets (here supposed separated into $\mathrm{M}$ ), each one with an angle width $\Delta \theta \Delta \varphi$, where $\Delta \varphi=$ $2 \pi / M$. Each facet contributes to the field on $\mathrm{Q}$, so according equation (1), the field on $\mathrm{Q}$ is

$$
\begin{aligned}
& \left.\tilde{E}\right|_{x}=\sum_{m=0}^{M} \tilde{C}_{1}(\theta) \cos (m \Delta \varphi) e^{i \Psi_{m}} \hat{x} \\
& \left.\tilde{E}\right|_{y}=\sum_{m=0}^{M} \tilde{C}_{1}(\theta) \sin (m \Delta \varphi) e^{i \Psi_{m}} \hat{y}
\end{aligned}
$$

where

$$
\begin{gathered}
\Psi_{m}=k \rho_{Q} \sin \theta \cos (m \Delta \varphi)=k_{0} \rho_{Q} N \cdot A \cdot \cos (m \Delta \varphi) \\
\tilde{C}_{1}(\theta)=\left(-i f A_{0} / \lambda\right)(\cos \theta)^{3 / 2} \sin \theta \Delta \theta \Delta \varphi
\end{gathered}
$$

$\Psi_{m}$ is a cosine function of $m \Delta \varphi$ with amplitude $k_{0} \rho_{0} N . A$. When $m$ changes from 0 to $M, \Psi_{m}$ gets all of its values in one cycle as shown in Figure 3.

By applying PI to describe formulas (2) and (3), Figure 4 is achieved. In Figure 4, on the curves, between each two neighbor points there is a small vector and each vector denotes the contribution of one small facet to the field on point $\mathrm{Q}$. The small vector's length and direction represent its amplitude and phase respectively. $\zeta$ and $\eta$ are the assistant phase reference axes, and $\zeta$-axis denotes zero phase, and all other direction angles of the small vectors to $\zeta$-axis denote their phase $\Psi \mathrm{m}$. There, the first and the last vectors represent respectively the contribution of point $\mathrm{P}$ with $\varphi=0$ and $\varphi_{M}=(M-1) \star 2 \pi / M$. All M vectors link together, starting from $\mathrm{A}$ and ending at $\mathrm{B}$, thus, $\overrightarrow{A B}_{X}$ denotes the total $x$ polarized component on Q-point generated by all facets of annular beams. The same argument is applied to the y-polarized component, and the final vector is $\overrightarrow{A B}_{y}$. 


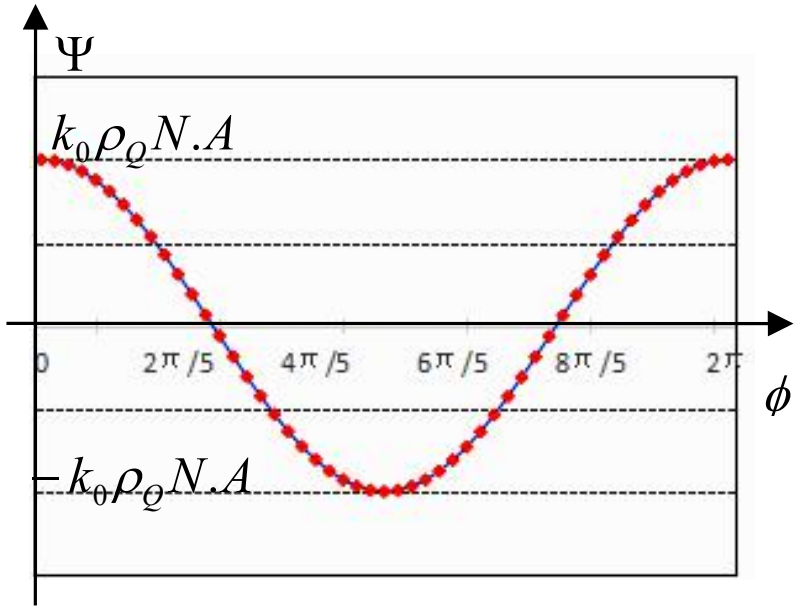

Figure 3: Phase values on point $Q$ generated by all small fifty facets on the annular beams. Phi is the azimuth of the facets, and $\Phi_{m}=$ $m \Delta \varphi$

Figure 4(a) shows PI curves on $\mathrm{Q}_{1}\left(\rho_{Q}=\lambda / 8, \varphi_{0}=\right.$ $\pi / 4)$, where $\overrightarrow{A B}_{x}=\overrightarrow{A B}_{y}$ indicates that the $\mathrm{x}$ and y components are equal, whether in amplitude or phase. Considering $\varphi_{0}=\pi / 4$, so the transverse field is radially polarized. For $\mathrm{Q}_{2}\left(\rho_{Q}=\lambda / 8, \varphi_{Q}=0\right)$, the PI curves are shown in Figure 4(b). Note that the PI curves of y component is a round trip, so the final result of the y component is zero, and only the $\mathrm{x}$ component exists. Considering $\varphi_{Q}=0$, so the $\mathrm{x}$ direction is just the radial direction, which is coincident with the result in Figure 4(a). The transverse field PI curves on different points are shown in Figure 4. There are 11 curves corresponding to 11 points $\left(\varphi_{Q i}=0, \rho_{Q i}=2(i-1) \lambda / 50\right.$, $\mathrm{i}=1,2, \ldots$. 11.), where the curves have been normalized by the maximum value. $\overrightarrow{A_{i} B_{i}}$ indicates the PI result on $Q_{i}$. On the z-axis $\left(\rho_{Q}=0\right), A_{1}$ overlaps with $B_{1}$, thus, $\overrightarrow{A_{1} B_{1}}=\overrightarrow{0}$, so the transverse field is zero. As $Q$ moves further away from the axis, the transverse field will increase and get its largest value at about $\rho_{Q i}=7 \lambda / 25$. When the annular beams' $N . A \neq 1$, the transverse field's maximum on the focal plane would locate at $\rho_{Q i}=(7 \lambda / 25) / N$.A. Figure 5 also indicates that the transverse field has a donut shape.

\subsection{Longitudinal focal properties}

According to formula (2), the longitudinal field of point $Q$ generated by all facets on annular beams can be written as

$$
\tilde{E}_{z}(\theta)=\sum_{m=0}^{M} \tilde{C}_{2}(\theta) e^{i \psi_{m}}
$$
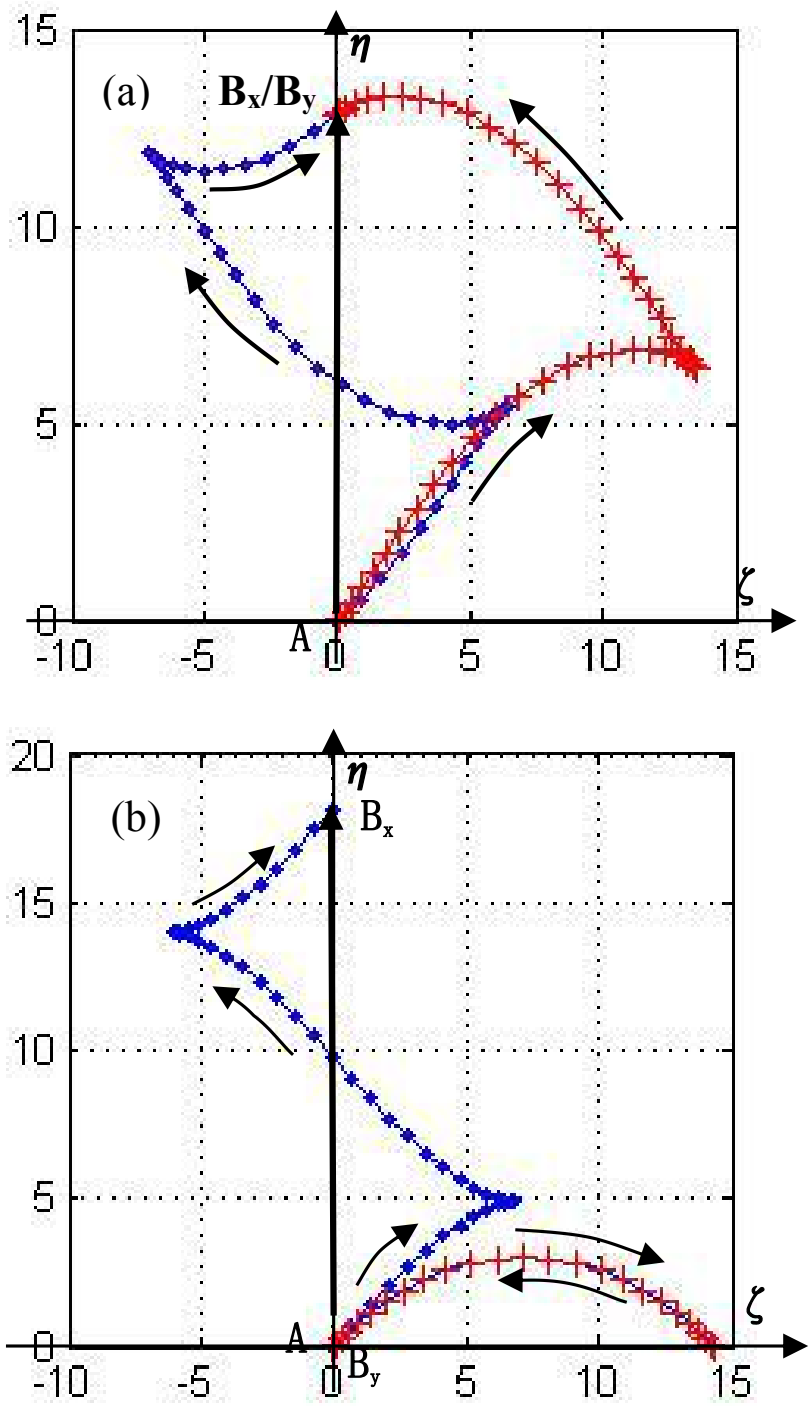

Figure 4: The PI curves of all small vectors generated by small facets on annular beams. The $\zeta$ axis indicates the direction of reference phase zero, and the $\eta$ axis indicates $\pi / 2$ relative phase. Here, all the values are relative to $\tilde{C}_{1}$. Blue “.”, red "*” for $x$ and y component respectively. ' $\rightarrow$ ' indicates the direction of superposition. $A$ is the beginning of the first small vector, and $\mathrm{B}_{x}, \mathrm{~B}_{y}$ are the end of the last small vector. (a) For $\Phi_{q}=\pi / 4$. (b) For $\Phi_{Q}=0$

where $\tilde{C}_{2}(\theta)=\left(-i f A_{0} / \lambda\right) \sqrt{\cos \theta} \sin ^{2} \theta \Delta \theta \Delta \varphi$, and the other parameters are same as those in formula (2). $\Psi_{m}$ still is same as described in Figure 2.

Using PI to express formula(6), we got Figure 6. There are eleven curves corresponding to eleven points $Q_{i}$ with $\varphi_{Q i}=0$ and $\rho_{Q_{j}}=(2 j-1) \lambda / 50, \mathrm{j}=1,2, \ldots .11$, and $\overrightarrow{A_{j} B_{j}}$ denotes the longitudinal field on $Q_{j}$. The trend of the curves indicates that the longitudinal field on the focus point is largest. When $Q$ is away from the axis the longitudinal field will decrease, and at $Q_{10}\left(\rho_{Q}=19 \lambda / 50\right)$ it will be zero. WithN.A $\neq 1$, the longitudinal field's zero on the focal 


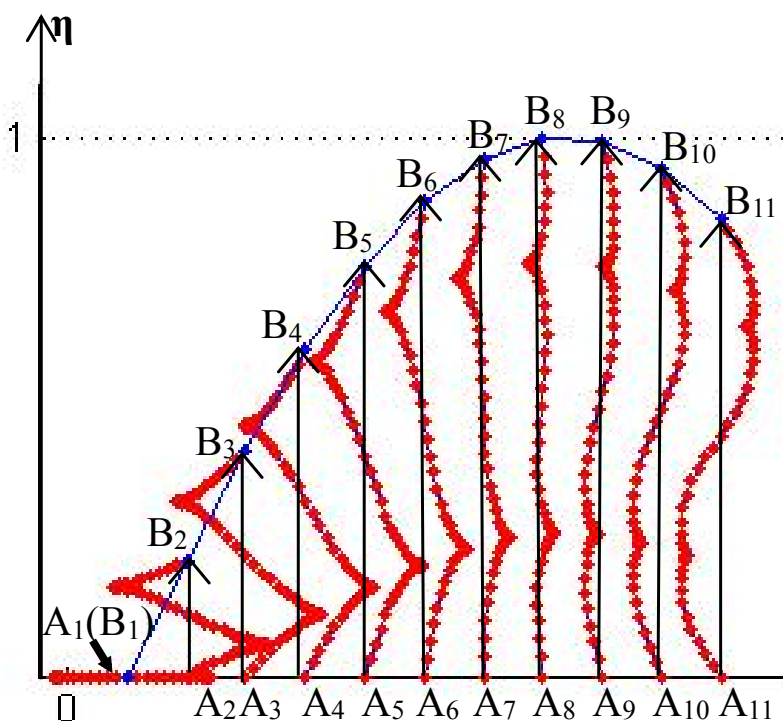

Figure 5: The transverse components $\mathrm{PI}$ curves generated by RP annular (N.A. $=1)$ beams on 11 points with $\varphi_{Q}=0, \rho_{Q_{i}}=2(i-1) \lambda / 50$, $j=1,2 \ldots 11$. All curves are normalized by the largest value

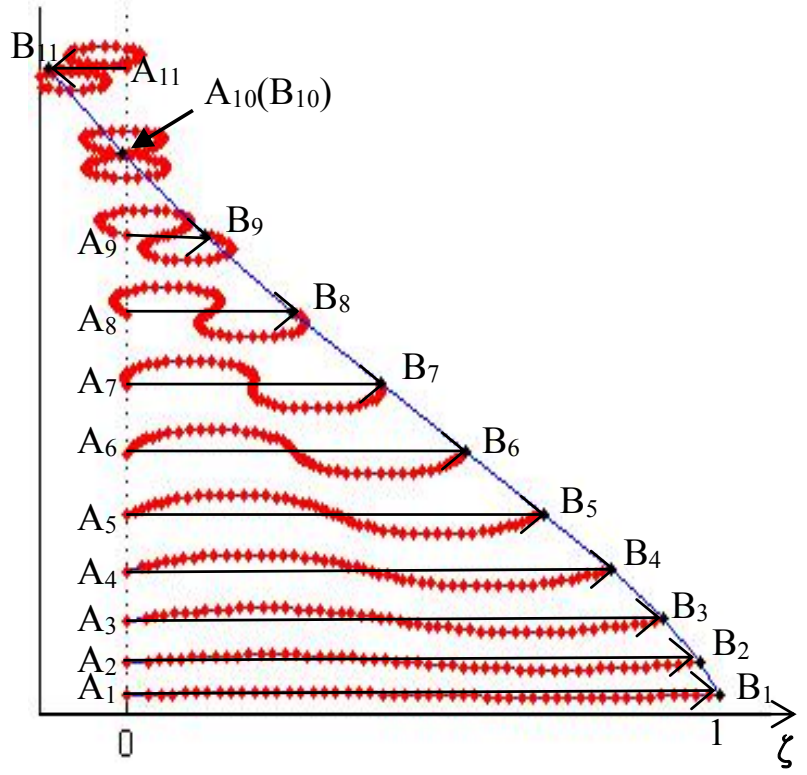

Figure 6: The longtitudinal component PI curves generated by RP annular beams (N.A=1). Corresponding to $\rho_{Q_{j}}=(2 j-1) \lambda / 50$ $\left(\mathrm{j}=1,2 \ldots\right.$.11) $\varphi_{Q}=0$ respectively, there are 11 curves. All curves are normalized by the largest value

plane locates at about $\rho_{Q}^{(0)}=19 \lambda /(50 N . A)$, which will be the Half Angle Line Width (HALW) of the longitudinal field. When the radial polarized annular beams is used as the illumination source of a focal microscopy, the HALW will determine the resolution, which is better than a traditional microscopy system's resolution of $0.61 \lambda / N$.A.

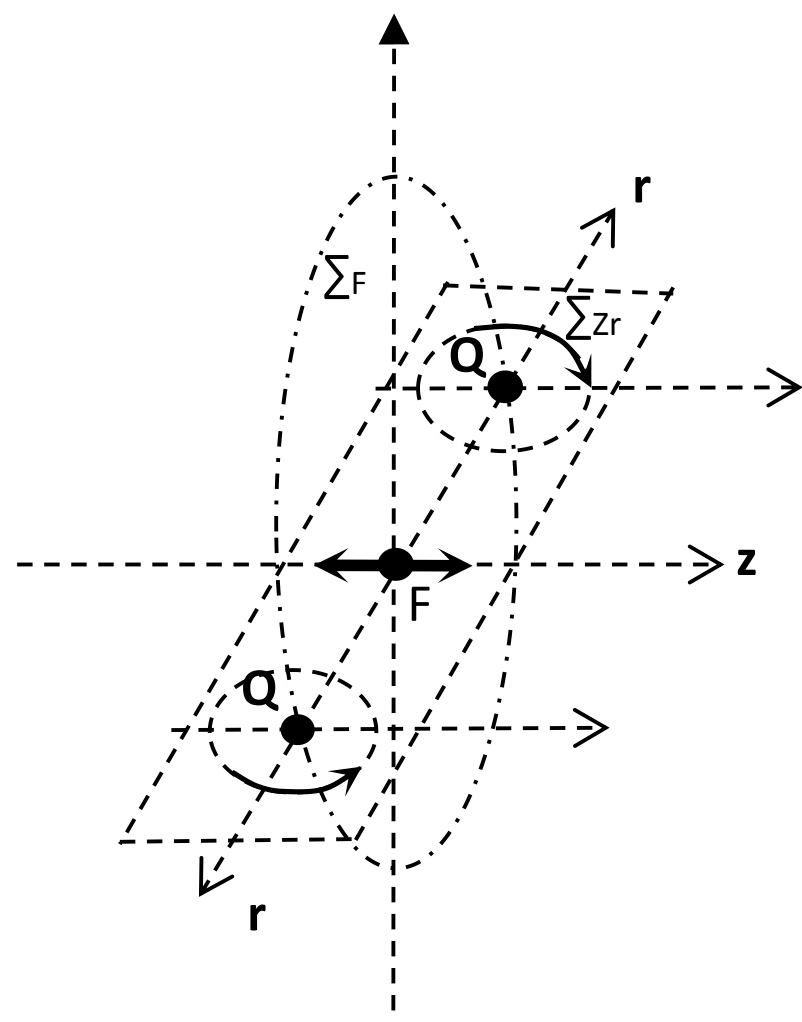

Figure 7: The polarization state distribution on the focal plane generated by RP annular beams. $\Sigma_{Z r}$ is the plane defined by $z$ and $r$. “ $\rightarrow$ " denotes the polarization direction

\subsection{Polarization state on the RP focal plane}

The polarization state of the total field is determined by the phase difference between the transverse and longitudinal fields. Firstly, Figure 5 indicates that there is no transverse field in the center, so the field on axis is linearly polarized along the z-axis. Secondly, for an off-axis point, the transverse vector is always along $\eta$, while the longitudinal vector is always along $\zeta$, so there is always $\pi / 2$ phase difference between them, so the field is elliptically polarized with $\boldsymbol{z}$ and $\boldsymbol{r}$ as the symmetry axis as shown in Figure 7, and the long and short axis of the ellipse are determined by the weights of the transverse and longitudinal components.

\section{AP beams focal properties}

Produced by annular beams with N.A $=1$ at $Q\left(\rho_{Q}=\lambda / 8\right.$, $\varphi_{Q}=0$ ) on the focal plane, the PI graphs of $\mathrm{x}$ and y polarized components are shown in Figure 8, where all values are relative to $\tilde{C}_{3}(\theta)=\left(-i f A_{0} / \lambda\right) \sqrt{\cos \theta} \sin \theta \Delta \theta \Delta \varphi$. Com- 


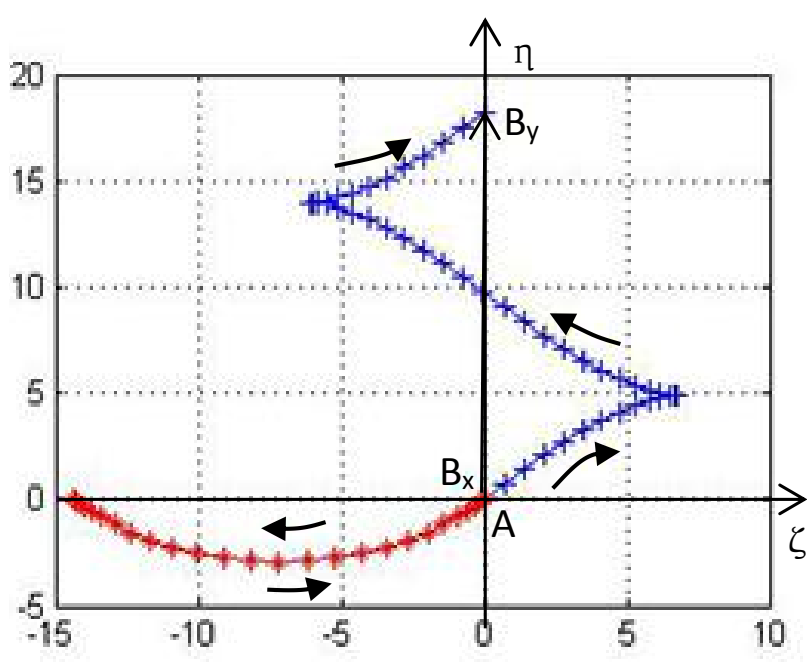

Figure 8: The PI curves on $Q\left(\rho_{Q}=\lambda / 8, \varphi_{Q}=0\right)$ produced by AP annular beams. Other parameters are the same as those in Figure 3. Here, the value is relative to $\tilde{C}_{3}(\theta)$

paring Figure 7 and Figure 8 we get

$$
\left\{\begin{array}{l}
\left.E_{X}\right|_{A P} \propto-\left.E_{y}\right|_{R P} \\
\left.E_{y} y\right|_{A P} \propto-\left.E_{x}\right|_{R P}
\end{array}\right.
$$

In fact, this relationship is coincident with Formula (1), only by considering $\cos \theta$ is a constant weight.

Figure 8 indicates that $\overrightarrow{A B_{x}}=0$, so there is only y polarized component on point $\mathrm{Q}$. Considering $\varphi_{Q}=0$, the $\mathrm{y}$ - component is along the azimuthal direction, so the AP beams' focal field is still azimuthally polarized.

Formula (1), Figure 4(b) and Figure 8 indicate that the focal field intensity of the AP beams is similar to the transverse component of RP beams', written as

$$
\left.I\right|_{A P}=\left.(1 / \cos \theta)^{2} I_{T}\right|_{R P}
$$

There for, the transverse component of RP beams' is donut on focal plane, so AP beam's focal field is donut too.

\section{Conclusion}

Figure 9 shows the transverse, longitudinal and total intensity distribution on focal plane generated by RP annular beams with N.A $=1$ (aperture angle $\theta=0.23 \pi$, index $n=1.5$ ), which is calculated by formula (1) using algebraic integral. It can be seen that the intensity distribution's characteristics obtained by PI, such as the field construction and the location of extreme points, are consistent with those obtained by pure algebraic integral, which shows the effectiveness of the PI method in analyzing the focusing of vector beams.
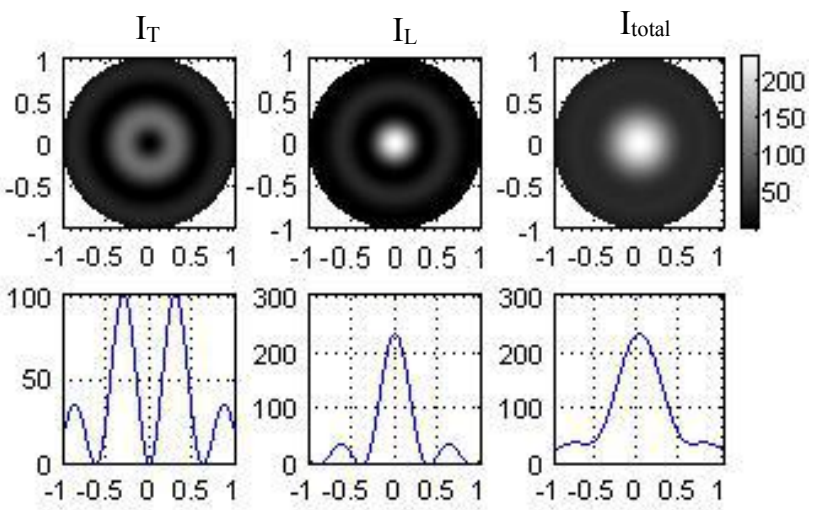

Figure 9: The transverse $\left(I_{T}\right)$, longitudinal $\left(I_{L}\right)$ and total $\left(I_{\text {total }}\right)$ intensity distribution on focal plane generated by RP annular beams with N.A $=1$ (aperture angle $\theta=0.23 \pi$, index $n=1.5$ ). Calculated by algebraic integral

But, in Figure 9, we only can see intensity distribution on focal plane, we can't see more detailed information, nor can we see the specific effect of each incident ray on the final results either. In contrast, PI has obvious advantages. More information of focal field can be extracted directly from PI curves. For example, from Figure 4, we can get the polarization state of the transverse field at the focal plane. While comparing Figure 4 and Figure 5, the phase difference between the transverse field and the longitudinal field can be obtained directly, then the total polarization state on the focal plane can be calculated. According to Figure 5, the RP beams' transverse field's doughnut structure can be predicted. By the changing trend of the PI curves in Figure 6, the position of the ' 0 ' field can be found. Figure 4(b) together with Figure 8 directly reveal the relationship between AP beams and transverse component of RP beams on focal plane. All above examples indicate that PI has more powerful information extraction function. Those obvious information in PI curves is easily submerged in the pure algebra integral calculating.

At the same time, PI also intuitively illustrate the effect of each part of the incident beam on the results, so when special focus field is needed, such as a tighter focusing, the use of PI will greatly improve the initiative in the designing of filter pupil.

Take the advantage of PI, even the focus field polarization distribution can be modulated, For example, according to Figure 4(b) and Figure 6, if half-wavelength filter is used in the half-space bounded by $\mathrm{x}$-axis, it is easy to conclude that the $\mathrm{x}$ and longitudinal components will be zero, while the original zero y-polarization component will no longer be zero, that means the field on the $\mathrm{x}$-axis will change from elliptical polarized to $y$-direction linear polarized light. So with the help of PI analysis, it's hopeful 
to design appropriate filter pupil to modulate light beams to meet different polarization requirements, which will be of great use in atomic capturing and manipulating, optical information coding, etc.

Acknowledgement: This work was supported by National Natural Science Foundation of China (Grant No. 61575067)

\section{References}

[1] Richards B., Wolf E. , Electromagnetic diffraction in optical systems II. Structure of the image field in an aplanatic system., Proc. Roy. Soc. A 1959, 253, 358-379.

[2] Youngworth K.S., Brown T. G., Focusing of high numerical aperture cylindrical-vector beams., Opt. Express, 2000, 7(2), 78-87.

[3] Quabis S., Dorn R., Eberler M., GlÖckl O., Leuchs G. , Focusing light to a tighter spot., Opt. Commun. 2000, 179, 1-7.

[4] Erdélyi M., Bor Zs., Radial and azimuthal polarizers., J.Opt.A, Pure Appl.Opt, 2006, 8, 737-742.

[5] Gilad M.L., Uriel L., Effect of radial polarization and apodization on spot size under tight focusing conditions., Opt. Express, 2008, 16(7),4567-4581.

[6] Suresh P., Mariyal C., Gokulakrishnan K., Rajesh K. B., Pillai T. V. S., Jaroszewicz Z., Investigation the focus shaping of the TEM11* beam with radial varying polarization., Optik, 2015, 126, 1691 1694.

[7] Li J., Feng K., Guo L., Phase filter design for sharper focus of radially polarized beam., Optik, 2014, 125, 3690-3692.

[8] Wang Y., Qiu L., Zhao W., High precision radially-polarized -light pupil-filtering differential confocal measurement., Opt. Laser Technol., 2016, 82, 87-93.

[9] Dorn R., Quabis S., Leuch G., Sharper Focus for a Radially Polarized Light Beam., Phs.Rev.Lett., 2003, 91(23), 233901, 1-4.

[10] Grosjean T., Suarez M., Sabac A., Generation of polychromatic radially and azimuthally polarized beams., Appl. Phys.Lett., 2008, 93, 231106.

[11] Hua Y., Wang Z., Li H., Nan G., Du Y., Radially and Azimuthally Polarized Beams Generated by a Composite Spiral Zone Plate., ChIN. PHYS. LETT., 2012, 29(8), 08214.
[12] Wang F., Zhao C., Dong Y., Generation and tight-focusing properties of cylindrical vector circular Airy beams., Appl.Phys.B., 2014, 117, 905-913.

[13] Wang X., Ren H., Nah Ch., Linearly to radially polarized light conversion and tight focus., J. Appl. Phys., 2015, 117, 243101.

[14] Kotlyar V. V., Nalimov A. G., Analytical expression for radiation forces on a dielectric cylinder illuminated by a cylindrical Gaussian beam., Opt. Express, 2006, 14, 6136-6321.

[15] Zhan Q., Trapping metallic Rayleigh particles with radial polarization., Opt. Express, 2004, 12(15), 3377-3382.

[16] Zhang Y., Ding B., Suyama T., Trapping two types of particles using a double-ring-shaped radially polarized beam., Phy. Rev. A, 2010, 81, 023831.

[17] Yan S., Yao B., Radiation forces of a highly focused radially polarized beam on spherical particles., Phy. Rev. A, 2007, 76, 053836.

[18] Kemepe M., Rudolph W., Analysis of confocal microscopy under ultrashort light-pulse illumination., J.Opt.Soc.Am.A., 1993, 10(2), 240-245.

[19] Gu M., Sheppard C. J. R., Analysis of confocal microscopy under ultrashort light-pulse illumination: comment, J.Opt.Soc.Am.A., 1994, 11(10), 2742-2473.

[20] Tang Z., Liang R., Chang H., The theory of two-photon confocal microscopy., Acta Phys. Sin-ch Ed., 2000, 49(6), 1076-1080.

[21] Tang Z., Yang C., Pei H., Liang R., Liu S., Imaging theory and resolution improvement of two-photon confocal microscopy., Sci. China. Ser. A, 2002, 45 (11), 1468-1478.

[22] Kim W.C., Park N.C., Yoon J.Y., Investigation of near-field imaging characteristics of radial polarization for application to optical data storage., Opt. Rev., 2007, 14(4), 236-242.

[23] Wei J. S., On the dynamic readout characteristic of nonlinear super-resolution optical storage., Opt. Commun., 2013, 291, 143149.

[24] Feynman R., QED, The strange theory of light and matter[M], (Chinese version., translated by Zhongjing Zhang), Commencial Press, 1996.

[25] Gokulakrishnan K., Suresh P., Mariyal C.,Sivasubramonia Pillai T. V., Rajesh K. B., Tight focusing effect of annular obstructed Bessel-modulated., Optik, 2014, 25, 6599-6601.

[26] Sheppard C. J. R., Choudbury A., Annular pupils, radial polarization, and supperesolution., Appl. Opt., 2004, 43(22), 4322-4327.

[27] Yang L., Xie X., Wang S., Zhou J., Minimized spot of annular radially Polarized focusing beam., Opt. Lett., 2013, 38(8),1331-1333. 